\title{
Better compliance with hypofractionation vs. conventional fractionation in adjuvant breast cancer radiotherapy
}

\author{
Results of a single, institutional, retrospective study \\ Volker Rudat $^{1} \cdot$ Alaa Nour $^{1} \cdot$ Mohamed Hammoud $^{1} \cdot$ Salam Abou Ghaida $^{1}$
}

Received: 13 November 2016 / Accepted: 7 February 2017 / Published online: 23 February 2017

(C) The Author(s) 2017. This article is available at SpringerLink with Open Access.

\begin{abstract}
Background The aim of the study was to identify factors significantly associated with the occurrence of unintended treatment interruptions in adjuvant breast cancer radiotherapy.

Patients and methods Patients treated with postoperative radiotherapy of the breast or chest wall between March 2014 and August 2016 were evaluated. The radiotherapy regimens and techniques applied were either conventional fractionation (CF; 28 daily fractions of $1.8 \mathrm{~Gy}$ or 25 fractions of $2.0 \mathrm{~Gy}$ ) or hypofractionation (HF; 15 daily fractions of $2.67 \mathrm{~Gy}$ ) with inverse planned intensity-modulated radiotherapy (IMRT) or three-dimensional planned conformal radiotherapy (3DCRT). Logistic regression analysis was used to identify factors associated with noncompliance. Noncompliance was defined as the missing of at least one scheduled radiotherapy fraction.

Results In all, 19 of 140 (13.6\%) patients treated with HF and 39 of $146(26.7 \%)$ treated with CF experienced treatment interruptions. Of 23 factors tested, the fractionation regimen emerged as the only independent significant prognostic factor for noncompliance on multivariate analysis (CF; $p=0.007$; odds ratio, $2.3 ; 95 \%$ confidence interval,
\end{abstract}

Prof. Dr. med. Volker Rudat

vrudat@saadmedical.com, volker.rudat@gmail.com

Alaa Nour

ANour@saadmedical.com

Mohamed Hammoud

MHammoud@saadmedical.com

Salam Abou Ghaida

SAbouGhaida@saadmedical.com

1 Department of Radiation Oncology, Saad Specialist Hospital, 31952 Al Khobar, Saudi Arabia
1.3-4.2). No statistically significant differences concerning the reasons for treatment interruptions could be detected between patients treated with CF or HF.

Conclusion HF is significantly associated with a better patient compliance with the prescribed radiotherapy schedule compared with CF. The data suggest that this finding is basically related to the shorter overall treatment time of HF.

Keywords Breast neoplasms - Radiotherapy - Dose hypofractionation $\cdot$ Radiation injuries $\cdot$ Risk factors

\section{Signifikant bessere Patientencompliance bei} hypofraktionierter im Vergleich zu konventionell fraktionierter adjuvanter Strahlentherapie des Mammakarzinoms

Ergebnisse einer unizentrischen retrospektiven Studie

\section{Zusammenfassung}

Hintergrund Ziel der Untersuchung war es, Faktoren zu identifizieren, die mit ungeplanten Behandlungsunterbrechungen bei der adjuvanten Strahlentherapie des Mammakarzinoms assoziiert sind.

Methoden und Patienten Es wurden Patienten untersucht, die eine adjuvante Strahlentherapie der Mamma oder Brustwand zwischen März 2014 und August 2016 erhielten. Zur Anwendung kamen als Fraktionierungsprotokoll und strahlentherapeutische Technik eine konventionell fraktionierte (CF; 28 Fraktionen mit 1,80 Gy oder 25 Fraktionen mit 2,00 Gy) oder eine hypofraktionierte Strahlentherapie (HF; 15 Fraktionen mit 2,67 Gy), eine intensitätsmodulierte (IMRT) oder dreidimensional geplante konformale Strahlentherapie (3DCRT). Mögliche mit Noncompliance assoziierte Faktoren wurden mittels logistischer Regressionsanalyse untersucht. Noncompliance wurde als gegeben be- 
trachtet bei dem Ausfall mindestens einer geplanten Strahlentherapiefraktion.

Ergebnisse Bei 19 von 140 (13,6\%) Patienten mit HF und 39 von $146(26,7 \%)$, die mit CF behandelt wurden, traten ungeplante Behandlungsunterbrechungen auf. Von 23 untersuchten Faktoren ging als einziger unabhängiger signifikanter Faktor für Noncompliance das Fraktionierungsprotokoll aus der multivariaten Analyse hervor (CF; $p=$ 0,007; Odds Ratio: 2,3; 95\%-Konfidenzintervall: 1,3-4,2). Bezüglich der Ursachen ungeplanter Behandlungsunterbrechungen konnte kein statistisch signifikanter Unterschied festgestellt werden.

Schlussfolgerung Die HF ist signifikant mit einer besseren Patientencompliance im Vergleich zur CF assoziiert. Die Daten legen nahe, dass diese Assoziation hauptsächlich auf die kürzere Gesamtbehandlungszeit der HF zurückzuführen ist.

Schlüsselwörter Neoplasien der Mamma •

Strahlentherapie $\cdot$ Hypofraktionierung $\cdot$ Strahlenbedingte

Nebenwirkungen $\cdot$ Risikofaktoren

\section{Background}

Unintended treatment interruptions may lead to a prolongation of the prescribed overall treatment time. For radiotherapy with curative intent, prolongation of the prescribed overall treatment time has been linked to inferior clinical outcomes [1-3]. This association appears to be consistent across many disease sites including head and neck cancer, cervical cancer, lung cancer, breast cancer, and other cancers $[4,5]$. Prospective and retrospective studies have shown that treatment prolongation can increase the risk of local recurrence by up to $2 \%$ per day for certain malignancies [5].

The association between prolongation of the prescribed overall treatment time and inferior clinical outcomes has been explained with an accelerated repopulation of tumor clonogens, which can occur after treatment initiation [6]. It has also been reported that noncompliance may serve as a behavioral biomarker for other risk factors that contribute to poor outcomes, such as noncompliance with other important clinician visits and procedures, lack of social support, and mood disorders [4].

In this study, we analyzed the compliance to the prescribed radiotherapy schedule of breast cancer patients treated with postoperative radiotherapy of the whole breast or chest wall. The goal of the study was to identify factors significantly associated with the occurrence of treatment interruptions.

\section{Patients and methods}

\section{Data collection and patient selection}

The electronic patient files of 286 consecutive unselected patients treated with adjuvant breast cancer radiotherapy between March 2014 and August 2016 were reviewed. Eligibility criteria for the analysis were (a) histologically proven diagnosis of breast cancer or carcinoma in situ and (b) treatment with adjuvant postoperative radiotherapy after breastconserving surgery or mastectomy. Exclusion criteria were bilateral breast cancer or history of previous radiotherapy of the breast or chest wall.

Patients were treated with either conventional fractionation (CF; 28 daily fractions of $1.8 \mathrm{~Gy}$ or 25 fractions of $2.0 \mathrm{~Gy}$ ) or hypofractionation (HF; 15 daily fractions of $2.67 \mathrm{~Gy}$ ). Where indicated, an electron boost was applied (five or eight daily fractions of $2.0 \mathrm{~Gy}$ ). Radiotherapy fractions were scheduled once per day and five times per week. Patients who missed radiotherapy fractions were offered to be treated on weekends in order not to exceed the prescribed overall treatment time. The radiation techniques used were inverse planned intensity-modulated radiotherapy (IMRT) or three-dimensional planned conformal radiotherapy using wedge compensation (3DCRT). The patients were thoroughly informed about the pros and cons of the two fractionation regimens and radiation techniques, and the treatment decision was mainly based on patient preference. Patients not covered, or not fully covered, by medical insurance tended to opt for 3DCRT for financial reasons. Patients with personal commitments limiting the overall treatment time or patients living far away from the radiotherapy facility tended to opt for HF.

The acute radiation reactions and reasons for treatment interruptions were documented prospectively in the Local Area Network Therapy Information System "Lantis" (Siemens Healthcare, Germany). The acute radiation reactions were assessed once weekly during radiotherapy and 6 weeks after radiotherapy by two observers using the Common Terminology Criteria for Adverse Events (CTCAE v4.03). The two observers were not involved in the statistical analysis of the study, and a table with all weekly assessments was included in the "End of Treatment Report" of all patients. The maximum acute radiation reaction observed during the full course of the radiotherapy (including the boost to the tumor bed if applied) was used for the statistical analysis. Treatment interruptions were defined as missing at least one of the scheduled daily radiotherapy fractions. The reasons for treatment interruptions were categorized into "public holidays," "patient unwillingness," "machine breakdown," "radiation reactions," and "unspecified," and documented prospectively together with the length of the treatment interruption. 
The study was approved by the local institutional ethics committee and conducted in accordance with the Helsinki Declaration in its current version.

\section{Treatment planning and radiation techniques}

The treatment planning and radiation techniques used for this study have been described in detail elsewhere [7-9]. In short, a non-contrast computed tomography (CT) simulation with a slice thickness of $5 \mathrm{~mm}$ was performed with the patient in the supine position. The planning target volume (PTV) of the whole breast or chest wall was defined according to the recommendations of the breast cancer atlas for radiation therapy planning consensus definitions of the Radiation Therapy Oncology Group (RTOG) [10]. The IMRT and 3DCRT plans were generated using the treatment planning system XIO 4.4 (CMS, Inc., St. Louis, Mo.). The dose to the PTV was prescribed according to the International Commission on Radiation Units and Measurement (ICRU) Reports 50 and 62 recommendations. Two Siemens Oncor Anvantgarde linear accelerators with a 160 MLC Multileaf Collimator were used for the treatment. Daily online verification and correction of the patient positioning error prior to radiotherapy were performed for all patients using orthogonal megavoltage electronic portal images [11]. No respiratory gating [12-14], integrated boost [15, 16], or partial breast irradiation [17] techniques were applied in this study. Two tangential semi-opposed beams, physical wedges (usually $15^{\circ}$ or $30^{\circ}$ ), a 160 MLC Multileaf Collimator and $6 \mathrm{MV}$ photons were used for the IMRT and 3DCRT plans. Occasionally a mixed-beam technique using $6 \mathrm{MV}$ and $15 \mathrm{MV}$ photons was used for the 3DCRT plans. Inverse treatment planning and a step-and-shoot technique were used for all IMRT plans. Tissue inhomogeneities were considered in the treatment planning optimization process, and the dose calculation algorithm used was "Superposition." A few patients with left-sided breast cancer and unfavorable thoracic geometry were treated with seven-field IMRT in order to reduce the high-dose region to the heart [18].

\section{Statistical analysis}

Differences between patient groups stratified by the occurrence of treatment interruptions (Table 1) or by the fractionation regimen (Table 3 ) were assessed using the chi-square test or $t$ test where appropriate. To assess the association of multiple factors with the occurrence of treatment interruptions, a univariate and multivariate logistic regression analysis was performed. The factors tested in the logistic regression analysis are listed in Table 2 . The model selection of the multivariate analysis was performed by a backward stepwise strategy. All tests were two-sided, and a $p$ value of $\leq 0.05$ was considered significant.

\section{Results}

In total, 58 of $286(20.3 \%)$ patients experienced treatment interruptions. The patient, disease, and treatment characteristics of the study population stratified by the occurrence of treatment interruptions are demonstrated in Table 1. As expected, the mean age of the study population was considerably lower compared with reports from Europe or the United States, most likely due to the young age structure of the general population [19].

On univariate analysis, three of 23 tested factors were significantly associated with a higher risk of treatment interruptions (Table 2). All three factors were related to longer treatment courses $(\mathrm{CF}$, number of radiotherapy fractions $\geq 29$, boost to the tumor bed). In total, 19 of $140(13.6 \%)$ patients treated with $\mathrm{HF}$ and 39 of 146 (26.7\%) treated with CF had treatment interruptions. On multivariate analysis, the only remaining independent significant prognostic factor was the fractionation regimen: CF vs. HF; $p=0.007$; odds ratio ( $95 \%$ confidence interval) $2.3(1.3,4.2)$.

Concerning the reasons for treatment interruptions, no statistically significant differences were detected between the patients treated with CF and HF (Table 3). However, treatment interruptions were on average longer for patients treated with CF (3.2 days vs. 2.3 days; $p=0.02$; Table 3).

In accordance with our departmental policy, treatment interruptions were compensated by treating the corresponding patients on weekends within the prescribed overall treatment time. After compensation for treatment interruptions, eventually $41.4 \%$ of the patients with treatment interruptions completed their treatment within the prescribed overall treatment time, corresponding to $88.1 \%$ of the total study population. The remaining patients experienced a prolongation of the prescribed overall treatment time of 1-5 days (Table 3).

\section{Discussion}

Our study shows that a significant proportion of our patients experienced unintended treatment interruptions (20.3\%). The compliance to the prescribed radiotherapy schedule was significantly better with HF than with $\mathrm{CF}$ (patients with treatment interruptions; $13.6 \%$ vs. $26.7 \%$ ). The data suggest that the better compliance was basically related to the shorter overall treatment time of HF (3-4 weeks) compared with CF (5-6.5 weeks).

Several randomized trials have shown that HF is equally effective in long-term disease control and late radiation ef- 
Table 1 Patient, disease, and treatment characteristics stratified by occurrence of treatment interruptions

\begin{tabular}{|c|c|c|c|c|c|c|c|c|}
\hline \multirow[t]{3}{*}{ Characteristics } & & \multicolumn{2}{|l|}{ Total } & \multicolumn{4}{|c|}{ Treatment interruptions } & \multirow[t]{3}{*}{$p$} \\
\hline & & \multirow[b]{2}{*}{$n$} & \multirow[b]{2}{*}{$\%$} & \multicolumn{2}{|l|}{ Yes } & \multicolumn{2}{|l|}{ No } & \\
\hline & & & & $n$ & $\%$ & $n$ & $\%$ & \\
\hline Patients & & 286 & 100 & 58 & 20.3 & 228 & 79.7 & - \\
\hline \multirow[t]{4}{*}{ Country of origin } & Middle East & 208 & 72.7 & 42 & 72.4 & 166 & 72.8 & 0.18 \\
\hline & Asia & 42 & 14.7 & 7 & 12.1 & 35 & 15.4 & - \\
\hline & Africa & 25 & 8.7 & 4 & 6.9 & 21 & 9.2 & - \\
\hline & Europe/USA & 11 & 3.8 & 5 & 8.6 & 6 & 2.6 & - \\
\hline Age at diagnosis (years) & Mean (SD) & 48 & (9.6) & 48 & $(8.9)$ & 49 & $(9.8)$ & $0.65^{\mathrm{a}}$ \\
\hline \multirow[t]{3}{*}{ Body mass index } & $<25$ & 47 & 16.5 & 12 & 20.7 & 35 & 15.4 & 0.57 \\
\hline & $25-29$ & 76 & 26.7 & 16 & 27.6 & 60 & 26.4 & - \\
\hline & $\geq 30$ & 162 & 56.8 & 30 & 51.7 & 132 & 58.1 & - \\
\hline \multirow[t]{2}{*}{ Menopausal status } & Premenopausal & 143 & 50.0 & 31 & 53.4 & 112 & 49.1 & 0.56 \\
\hline & Postmenopausal & 143 & 50.0 & 27 & 46.6 & 116 & 50.9 & - \\
\hline \multirow[t]{2}{*}{ Marital status } & Married & 270 & 94.4 & 57 & 98.3 & 213 & 93.4 & 0.15 \\
\hline & Single & 16 & 5.6 & 1 & 1.7 & 15 & 6.6 & - \\
\hline \multirow[t]{2}{*}{ Financial status } & Medical insurance & 163 & 57.0 & 30 & 51.7 & 133 & 58.3 & 0.36 \\
\hline & Cash & 123 & 43.0 & 28 & 48.3 & 95 & 41.7 & - \\
\hline \multirow{3}{*}{$\begin{array}{l}\text { Distance from home to treatment facility } \\
(\mathrm{km})\end{array}$} & $\leq 50$ & 183 & 64.0 & 39 & 67.2 & 144 & 63.2 & 0.65 \\
\hline & $51-100$ & 62 & 21.7 & 10 & 17.2 & 52 & 22.8 & - \\
\hline & $>100$ & 41 & 14.3 & 9 & 15.5 & 32 & 14.0 & - \\
\hline \multirow[t]{4}{*}{ Pathohistology } & Invasive ductal cancer & 264 & 92.3 & 53 & 91.4 & 211 & 92.5 & 0.83 \\
\hline & $\begin{array}{l}\text { Invasive lobular can- } \\
\text { cer }\end{array}$ & 15 & 5.2 & 4 & 6.9 & 11 & 4.8 & - \\
\hline & DCIS & 5 & 1.7 & 1 & 1.7 & 4 & 1.8 & - \\
\hline & Other & 2 & 0.7 & 0 & 0.0 & 2 & 0.9 & - \\
\hline \multirow[t]{4}{*}{ Grading } & G1 & 22 & 7.7 & 6 & 10.3 & 16 & 7.0 & 0.31 \\
\hline & $\mathrm{G} 2$ & 91 & 31.8 & 20 & 34.5 & 71 & 31.1 & - \\
\hline & G3 & 146 & 51.0 & 24 & 41.4 & 122 & 53.5 & - \\
\hline & Not reported & 27 & 9.4 & 8 & 13.8 & 19 & 8.3 & - \\
\hline \multirow[t]{7}{*}{$\mathrm{T}$ classification } & pTis & 6 & 2.1 & 2 & 3.4 & 4 & 1.8 & 0.92 \\
\hline & pT0 & 8 & 2.8 & 2 & 3.4 & 6 & 2.6 & - \\
\hline & pT1 & 98 & 34.3 & 22 & 37.9 & 76 & 33.3 & - \\
\hline & pT2 & 114 & 39.9 & 22 & 37.9 & 92 & 40.4 & - \\
\hline & pT3 & 30 & 10.5 & 4 & 6.9 & 26 & 11.4 & - \\
\hline & pT4 & 20 & 7.0 & 4 & 6.9 & 16 & 7.0 & - \\
\hline & Not reported & 10 & 3.5 & 2 & 3.4 & 8 & 3.5 & - \\
\hline \multirow[t]{5}{*}{$\mathrm{N}$ classification } & pNO & 102 & 35.7 & 28 & 48.3 & 74 & 32.5 & 0.20 \\
\hline & $\mathrm{pN} 1$ & 84 & 29.4 & 13 & 22.4 & 71 & 31.1 & - \\
\hline & $\mathrm{pN} 2$ & 60 & 21.0 & 12 & 20.7 & 48 & 21.1 & - \\
\hline & $\mathrm{pN} 3$ & 33 & 11.5 & 4 & 6.9 & 29 & 12.7 & - \\
\hline & Not reported & 7 & 2.4 & 1 & 1.7 & 6 & 2.6 & - \\
\hline \multirow[t]{2}{*}{ M classification } & $\mathrm{cM} 0$ & 282 & 98.6 & 58 & 100.0 & 224 & 98.2 & 0.31 \\
\hline & $\mathrm{cM} 1$ & 4 & 1.4 & 0 & 0.0 & 4 & 1.8 & - \\
\hline ER status & Positive & 204 & 71.3 & 43 & 74.1 & 161 & 70.6 & 0.62 \\
\hline & Negative & 71 & 24.8 & 14 & 24.1 & 57 & 25.0 & - \\
\hline & Not reported & 11 & 3.8 & 1 & 1.7 & 10 & 4.4 & - \\
\hline PR status & Positive & 184 & 64.3 & 37 & 63.8 & 147 & 64.5 & 0.89 \\
\hline & Negative & 86 & 30.1 & 17 & 29.3 & 69 & 30.3 & - \\
\hline & Not reported & 16 & 5.6 & 4 & 6.9 & 12 & 5.3 & - \\
\hline Her2/neu status & Positive & 79 & 27.6 & 17 & 29.3 & 62 & 27.2 & 0.94 \\
\hline & Negative & 188 & 65.7 & 37 & 63.8 & 151 & 66.2 & - \\
\hline
\end{tabular}


Table 1 Patient, disease, and treatment characteristics stratified by occurrence of treatment interruptions (Continued)

\begin{tabular}{|c|c|c|c|c|c|c|c|c|}
\hline \multirow[t]{3}{*}{ Characteristics } & & \multicolumn{2}{|c|}{ Total } & \multicolumn{4}{|c|}{ Treatment interruptions } & \multirow[t]{3}{*}{$p$} \\
\hline & & \multirow[b]{2}{*}{$n$} & \multirow[b]{2}{*}{$\%$} & \multicolumn{2}{|l|}{ Yes } & \multicolumn{2}{|l|}{ No } & \\
\hline & & & & $n$ & $\%$ & $n$ & $\%$ & \\
\hline \multirow{3}{*}{ Planning target volume (PTV) } & Not reported & 19 & 6.6 & 4 & 6.9 & 15 & 6.6 & - \\
\hline & Chest wall & 150 & 52.4 & 27 & 46.6 & 123 & 53.9 & 0.31 \\
\hline & Whole breast & 136 & 47.6 & 31 & 53.4 & 105 & 46.1 & - \\
\hline \multirow[t]{4}{*}{ Volume of PTV $\left(\mathrm{cm}^{3}\right)$} & $\leq 652$ & 71 & 24.8 & 9 & 15.5 & 62 & 27.2 & 0.33 \\
\hline & $653-872$ & 72 & 25.2 & 17 & 29.3 & 55 & 24.1 & - \\
\hline & $873-1235$ & 71 & 24.8 & 16 & 27.6 & 55 & 24.1 & - \\
\hline & $\geq 1236$ & 72 & 25.2 & 16 & 27.6 & 56 & 24.6 & - \\
\hline \multirow{2}{*}{$\begin{array}{l}\text { Locoregional lymph nodes treated as part } \\
\text { of plan }\end{array}$} & Yes & 149 & 52.1 & 25 & 43.1 & 124 & 54.4 & 0.12 \\
\hline & No & 137 & 47.9 & 33 & 56.9 & 104 & 45.6 & - \\
\hline \multirow[t]{2}{*}{ Boost to the tumor bed } & Yes & 133 & 46.5 & 34 & 58.6 & 99 & 43.4 & 0.04 \\
\hline & No & 153 & 53.5 & 24 & 41.4 & 129 & 56.6 & - \\
\hline \multirow[t]{2}{*}{ Radiotherapy technique } & TB-IMRT & 167 & 58.4 & 33 & 56.9 & 134 & 58.8 & 0.80 \\
\hline & 3DCRT & 119 & 41.6 & 25 & 43.1 & 94 & 41.2 & - \\
\hline \multirow[t]{2}{*}{ Fractionation regimen } & $\mathrm{CF}$ & 146 & 51.0 & 39 & 67.2 & 107 & 46.9 & 0.01 \\
\hline & $\mathrm{HF}$ & 140 & 49.0 & 19 & 32.8 & 121 & 53.1 & - \\
\hline \multirow[t]{4}{*}{ Number of fractions } & $\leq 15$ & 86 & 30.1 & 11 & 19.0 & 75 & 32.9 & 0.01 \\
\hline & $16-20$ & 45 & 15.7 & 8 & 13.8 & 37 & 16.2 & - \\
\hline & $21-28$ & 70 & 24.5 & 11 & 19.0 & 59 & 25.9 & - \\
\hline & $\geq 29$ & 85 & 29.7 & 28 & 48.3 & 57 & 25.0 & - \\
\hline \multirow[t]{3}{*}{ Chemotherapy } & Adjuvant & 211 & 73.8 & 41 & 70.7 & 170 & 74.6 & 0.58 \\
\hline & Neo-adjuvant & 61 & 21.3 & 15 & 25.9 & 46 & 20.2 & - \\
\hline & No chemotherapy & 14 & 4.9 & 2 & 3.4 & 12 & 5.3 & - \\
\hline \multirow[t]{2}{*}{ Hormone therapy } & Yes & 210 & 73.4 & 44 & 75.9 & 166 & 72.8 & 0.64 \\
\hline & No & 76 & 26.6 & 14 & 24.1 & 62 & 27.2 & - \\
\hline \multirow[t]{3}{*}{ Fatigue (grade CTCAE v4.0) } & 0 & 101 & 35.3 & 24 & 41.4 & 77 & 33.8 & 0.51 \\
\hline & 1 & 172 & 60.1 & 31 & 53.4 & 141 & 61.8 & - \\
\hline & 2 & 13 & 4.5 & 3 & 5.2 & 10 & 4.4 & - \\
\hline \multirow[t]{4}{*}{ Dermatitis radiation (grade CTCAE v4.0) } & 0 & 11 & 3.8 & 2 & 3.4 & 9 & 3.9 & 0.37 \\
\hline & 1 & 228 & 79.7 & 42 & 72.4 & 186 & 81.6 & - \\
\hline & 2 & 44 & 15.4 & 13 & 22.4 & 31 & 13.6 & - \\
\hline & 3 & 3 & 1.0 & 1 & 1.7 & 2 & 0.9 & - \\
\hline \multirow[t]{3}{*}{ Dysphagia (grade CTCAE v4.0) } & 0 & 207 & 72.4 & 47 & 81.0 & 160 & 70.2 & 0.26 \\
\hline & 1 & 72 & 25.2 & 10 & 17.2 & 62 & 27.2 & - \\
\hline & 2 & 7 & 2.4 & 1 & 1.7 & 6 & 2.6 & - \\
\hline \multirow[t]{3}{*}{ Esophagitis (grade CTCAE v4.0) } & 0 & 265 & 92.7 & 51 & 87.9 & 214 & 93.9 & 0.21 \\
\hline & 1 & 20 & 7.0 & 7 & 12.1 & 13 & 5.7 & - \\
\hline & 2 & 1 & 0.3 & 0 & 0.0 & 1 & 0.4 & - \\
\hline \multirow[t]{3}{*}{ Cough (grade CTCAE v4.0) } & 0 & 266 & 93.0 & 54 & 93.1 & 212 & 93.0 & 0.65 \\
\hline & 1 & 17 & 5.9 & 4 & 6.9 & 13 & 5.7 & - \\
\hline & 2 & 3 & 1.0 & 0 & 0.0 & 3 & 1.3 & - \\
\hline Dyspnea (grade CTCAE v4.0) & 0 & 277 & 96.9 & 56 & 96.6 & 221 & 96.9 & 0.83 \\
\hline & 1 & 8 & 2.8 & 2 & 3.4 & 6 & 2.6 & - \\
\hline & 2 & 1 & 0.3 & 0 & 0.0 & 1 & 0.4 & - \\
\hline
\end{tabular}

$p$ Values using chi-square testing to compare patient subgroups with and without treatment interruptions, except as indicated

$D C I S$ ductal carcinoma in situ, $E R$ estrogen receptor, $P R$ progesterone receptor, TB-IMRT tangential beam intensity-modulated radiotherapy, $3 D C R T$ three-dimensional conformal radiotherapy, $C F$ conventional fractionation, $H F$ hypofractionation, $C T C A E$ Common Terminology Criteria for Adverse Events

a'Unpaired Student's $t$ test 
Table 2 Univariate logistic regression results for associations with treatment interruptions

\begin{tabular}{|c|c|c|c|c|c|}
\hline Characteristics & & $\begin{array}{l}\text { Odds } \\
\text { ratio }\end{array}$ & $\begin{array}{l}\text { Lower } \\
95 \% \mathrm{CI} \\
\end{array}$ & $\begin{array}{l}\text { Upper } \\
95 \% \mathrm{CI} \\
\end{array}$ & $p$ \\
\hline \multirow[t]{4}{*}{ Country of origin } & Middle East & Reference & & & \\
\hline & Asia & 1.33 & 0.43 & 4.08 & 0.62 \\
\hline & Africa & 1.27 & 0.53 & 3.05 & 0.60 \\
\hline & Europe/USA & 0.30 & 0.09 & 1.04 & 0.06 \\
\hline \multirow[t]{2}{*}{ Age at diagnosis (years) } & $\leq$ Mean & Reference & & & \\
\hline & $>$ Mean & 0.92 & 0.51 & 1.64 & 0.77 \\
\hline \multirow[t]{3}{*}{ Body mass index } & $<25$ & Reference & & & \\
\hline & $25-29$ & 1.29 & 0.55 & 3.03 & 0.57 \\
\hline & $\geq 30$ & 1.51 & 0.70 & 3.25 & 0.29 \\
\hline \multirow[t]{2}{*}{ Menopausal status } & Premenopausal & Reference & & & \\
\hline & Postmenopausal & 1.19 & 0.67 & 2.12 & 0.56 \\
\hline \multirow[t]{2}{*}{ Marital status } & Married & Reference & & & \\
\hline & Single & 0.25 & 0.03 & 1.93 & 0.18 \\
\hline \multirow[t]{2}{*}{ Financial status } & Medical insurance & Reference & & & \\
\hline & Cash payer & 0.77 & 0.43 & 1.36 & 0.36 \\
\hline \multirow[t]{3}{*}{ Commuting distance to treatment facility $(\mathrm{km})$} & $\leq 50$ & Reference & & & \\
\hline & $51-100$ & 1.04 & 0.46 & 2.36 & 0.93 \\
\hline & $>100$ & 1.46 & 0.54 & 3.99 & 0.46 \\
\hline \multirow[t]{2}{*}{ Planning target volume (PTV) } & Chest wall & Reference & & & \\
\hline & Whole breast & 0.74 & 0.42 & 1.33 & 0.31 \\
\hline \multirow[t]{4}{*}{ Volume of PTV $\left(\mathrm{cm}^{3}\right)$} & $\leq 652$ & Reference & & & \\
\hline & $653-872$ & 0.47 & 0.19 & 1.14 & 0.09 \\
\hline & $873-1235$ & 0.50 & 0.20 & 1.22 & 0.13 \\
\hline & $\geq 1236$ & 0.51 & 0.21 & 1.24 & 0.14 \\
\hline \multirow[t]{2}{*}{ Locoregional lymph nodes treated as part of plan } & Yes & Reference & & & \\
\hline & No & 0.64 & 0.36 & 1.14 & 0.13 \\
\hline \multirow[t]{2}{*}{ Boost to the tumor bed } & Yes & Reference & & & \\
\hline & No & 1.85 & 1.03 & 3.31 & 0.04 \\
\hline \multirow[t]{2}{*}{ Radiotherapy technique } & IMRT & Reference & & & \\
\hline & 3DCRT & 0.93 & 0.52 & 1.66 & 0.80 \\
\hline \multirow[t]{2}{*}{ Fractionation regimen } & $\mathrm{CF}$ & Reference & & & \\
\hline & $\mathrm{HF}$ & 2.32 & 1.27 & 4.26 & $<0.01$ \\
\hline \multirow[t]{4}{*}{ Number of radiotherapy fractions } & $\leq 15$ & Reference & & & \\
\hline & $16-20$ & 0.68 & 0.25 & 1.83 & 0.44 \\
\hline & $21-28$ & 0.79 & 0.32 & 1.94 & 0.60 \\
\hline & $\geq 29$ & 0.30 & 0.14 & 0.65 & $<0.01$ \\
\hline \multirow[t]{3}{*}{ Chemotherapy } & Adjuvant & Reference & & & \\
\hline & Neo-adjuvant & 0.74 & 0.38 & 1.45 & 0.38 \\
\hline & No chemotherapy & 1.45 & 0.31 & 6.72 & 0.64 \\
\hline \multirow[t]{2}{*}{ Hormone therapy } & Yes & Reference & & & \\
\hline & No & 1.25 & 0.64 & 2.44 & 0.51 \\
\hline \multirow[t]{2}{*}{ Fatigue (grade CTCAE v4.0) } & 0 & Reference & & & \\
\hline & $>0$ & 0.74 & 0.41 & 1.33 & 0.31 \\
\hline \multirow[t]{2}{*}{ Dermatitis radiation (grade CTCAE v4.0) } & 0 & Reference & & & \\
\hline & $>0$ & 1.15 & 0.24 & 5.48 & 0.86 \\
\hline \multirow[t]{2}{*}{ Dysphagia (grade CTCAE v4.0) } & 0 & Reference & & & \\
\hline & $>0$ & 0.55 & 0.27 & 1.13 & 0.10 \\
\hline
\end{tabular}


Table 2 Univariate logistic regression results for associations with treatment interruptions (Continued)

\begin{tabular}{|c|c|c|c|c|c|}
\hline \multicolumn{2}{|l|}{ Characteristics } & \multirow{2}{*}{$\begin{array}{l}\begin{array}{l}\text { Odds } \\
\text { ratio }\end{array} \\
\text { Refere }\end{array}$} & \multirow{2}{*}{$\begin{array}{l}\text { Lower } \\
95 \% \mathrm{CI}\end{array}$} & \multirow{2}{*}{$\begin{array}{l}\text { Upper } \\
95 \% \mathrm{CI}\end{array}$} & \multirow[t]{2}{*}{$p$} \\
\hline Esophagitis (grade CTCAE v4.0) & 0 & & & & \\
\hline & $>0$ & 2.10 & 0.81 & 5.46 & 0.13 \\
\hline \multirow[t]{2}{*}{ Cough (grade CTCAE v4.0) } & 0 & Refer & & & \\
\hline & $>0$ & 0.98 & 0.32 & 3.06 & 0.97 \\
\hline \multirow[t]{2}{*}{ Dyspnea (grade CTCAE v4.0) } & 0 & Refer & & & \\
\hline & $>0$ & 1.13 & 0.23 & 5.58 & 0.88 \\
\hline \multirow[t]{2}{*}{ Any acute radiation reaction (grade CTCAE v4.0) } & 0,1 & Refer & & & \\
\hline & 2,3 & 1.69 & 0.87 & 3.28 & 0.12 \\
\hline
\end{tabular}

$C I$ confidence interval, IMRT intensity-modulated radiotherapy, $3 D C R T$ three-dimensional conformal radiotherapy, $C F$ conventional fractionation, $H F$ hypofractionation, CTCAE Common Terminology Criteria for Adverse Events

Table 3 Reason for and length of treatment interruptions stratified by fractionation regimen

\begin{tabular}{|c|c|c|c|c|c|c|c|c|}
\hline \multicolumn{2}{|c|}{ Reason for treatment interruptions } & \multicolumn{2}{|c|}{ Total } & \multicolumn{4}{|c|}{ Fractionation regimen } & \multirow[t]{3}{*}{$p$} \\
\hline & & \multirow[b]{2}{*}{$n$} & \multirow[b]{2}{*}{$\%$} & \multicolumn{2}{|l|}{$\mathrm{HF}$} & \multicolumn{2}{|l|}{$\mathrm{CF}$} & \\
\hline & & & & $n$ & $\%$ & $n$ & $\%$ & \\
\hline \multirow[t]{3}{*}{ Public holidays } & Yes & 33 & 56.9 & 11 & 57.9 & 22 & 56.4 & 0.91 \\
\hline & No & 25 & 43.1 & 8 & 42.1 & 17 & 43.6 & - \\
\hline & Days, mean (SD) & 1.9 & $(1.8)$ & 1.6 & $(1.5)$ & 2.1 & $(2.0)$ & $0.34^{\mathrm{a}}$ \\
\hline \multirow{3}{*}{$\begin{array}{l}\text { Patient } \\
\text { unwillingness }\end{array}$} & Yes & 23 & 39.7 & 8 & 42.1 & 15 & 38.5 & 0.79 \\
\hline & No & 35 & 60.3 & 11 & 57.9 & 24 & 61.5 & - \\
\hline & Days, mean (SD) & 0.6 & $(0.9)$ & 0.5 & $(0.7)$ & 0.6 & $(0.9)$ & $0.80^{\mathrm{a}}$ \\
\hline \multirow{3}{*}{$\begin{array}{l}\text { Machine } \\
\text { breakdown }\end{array}$} & Yes & 8 & 13.8 & 1 & 5.3 & 7 & 17.9 & 0.19 \\
\hline & No & 50 & 86.2 & 18 & 94.7 & 32 & 82.1 & - \\
\hline & Days, mean (SD) & 0.3 & $(0.8)$ & 0.1 & $(0.2)$ & 0.4 & $(1.0)$ & $0.16^{\mathrm{a}}$ \\
\hline \multirow{3}{*}{$\begin{array}{l}\text { Radiation } \\
\text { reactions }\end{array}$} & Yes & 4 & 6.9 & 0 & 0 & 4 & 10.3 & 0.15 \\
\hline & No & 54 & 93.1 & 19 & 100 & 35 & 89.7 & - \\
\hline & Days, mean (SD) & 0.1 & $(0.3)$ & 0 & $(0)$ & 0.1 & $(0.3)$ & $0.16^{\mathrm{a}}$ \\
\hline \multirow[t]{3}{*}{ Unspecified } & Yes & 1 & 1.7 & 1 & 5.3 & 0 & 0 & 0.15 \\
\hline & No & 57 & 98.3 & 18 & 94.7 & 39 & 100 & - \\
\hline & Days, mean (SD) & 0.1 & $(0.4)$ & 0.2 & $(0.7)$ & 0 & $(0)$ & $0.15^{\mathrm{a}}$ \\
\hline \multirow{5}{*}{$\begin{array}{l}\text { Treatment } \\
\text { interruptions }\end{array}$} & Yes & 58 & 20.3 & 19 & 13.6 & 39 & 26.7 & 0.01 \\
\hline & No & 228 & 79.7 & 121 & 86.4 & 107 & 73.3 & - \\
\hline & For one reason & 47 & 81.0 & 17 & 89.5 & 30 & 76.9 & 0.25 \\
\hline & For two reasons & 11 & 19.0 & 2 & 10.5 & 9 & 23.1 & - \\
\hline & Days, mean (SD) & 2.9 & (1.4) & 2.3 & $(1.2)$ & 3.2 & (1.4) & $0.02^{\mathrm{a}}$ \\
\hline \multirow{6}{*}{$\begin{array}{l}\text { Prolongation of } \\
\text { the prescribed } \\
\text { overall treatment } \\
\text { time after } \\
\text { compensation for } \\
\text { treatment } \\
\text { interruptions }\end{array}$} & 0 days & 24 & 41.4 & 9 & 47.4 & 15 & 38.5 & 0.68 \\
\hline & 1 day & 15 & 25.9 & 6 & 31.6 & 9 & 23.1 & - \\
\hline & 2 days & 9 & 15.5 & 1 & 5.3 & 8 & 20.5 & - \\
\hline & 3 days & 5 & 8.6 & 1 & 5.3 & 4 & 10.3 & - \\
\hline & 4 days & 3 & 5.2 & 1 & 5.3 & 2 & 5.1 & - \\
\hline & 5 days & 2 & 3.4 & 1 & 5.3 & 1 & 2.6 & - \\
\hline
\end{tabular}

$p$ Values using chi-square testing to compare patient subgroups treated with $\mathrm{HF}$ or $\mathrm{CF}$, except as indicated

$C F$ conventional fractionation, $H F$ hypofractionation

a'Unpaired Student's $t$ test 
fects compared with CF in adjuvant breast cancer radiotherapy [20-23]. The main motivation for developing protracted radiotherapy regimens was the benefit to patients and health services in terms of convenience and cost. Recent breast cancer studies suggested that $\mathrm{HF}$ is also associated with a significantly lower acute skin reaction rate compared with CF $[7,24,25]$. Our study revealed another advantage of HF over CF: a significantly better patient compliance with the prescribed radiotherapy schedule.

Noncompliance with the prescribed radiotherapy schedule can have multiple deleterious effects. For postoperative radiotherapy of breast cancer, a prolongation of the overall treatment time of more than 1 week has been shown to decrease the 5 -year local control rate by $5 \%$ [26]. The management of the increased number of recurrences may place additional burden on the health-care system. Disturbances in the clinical workflow by noncompliant ("no-show") patients occupying treatment slots on the linear accelerator may indirectly cause treatment delays for other patients and an extension of the work day. Compensation of missed radiotherapy fractions during the working week by additional treatment on weekends will further increase costs in terms of time and effort.

In a large study of 2184 patients receiving radiotherapy with curative intent for various malignancies in an American urban academic cancer center, 20.2\% missed multiple radiotherapy fractions, $17.4 \%$ a single radiotherapy fraction, and $62.4 \%$ no radiotherapy fractions. The median number of missed treatments was 3. Similar to our study, the statistical analysis identified "prescribed longer radiotherapy courses" as a statistically significant independent predictor of noncompliance. The authors suggested that this finding may provide additional rationale for adopting shortened radiotherapy schedules as a means of improving patient adherence to prescribed therapy [27]. Other predictors for noncompliance identified in the previously cited study were "particular cancer diagnoses," "low socioeconomic status," and "treatment during winter months." "Distance from the patients' home to the radiotherapy facility" [28-30] and "patients from households that lost family income" [31] have been reported as predictors of noncompliance with the prescribed radiotherapy schedule by other study groups. It is likely that factors influencing compliance depend to a significant extent on individual circumstances like the location of the radiotherapy facility, infrastructure of the region, and socioeconomic status of the population, and may therefore vary between treatment facilities. However, in our study $\mathrm{CF}$, which was the longer radiotherapy schedule compared with HF, was the only significant predictor of noncompliance on multivariate analysis of 23 factors.

The limitations of our study should be noted. Owing to the relatively limited patient number $(n=286)$, possible influencing factors may not have reached statistical significance. The socioeconomic and psycho-oncological status of the patients could not be evaluated because of lack of data. Moreover, owing to the retrospective nature of the study, a selection bias of patients treated with HF and CF cannot be excluded with certainty.

Despite all efforts to avoid a prolongation of the prescribed overall treatment time by thorough education of the patient and compensation of missed radiotherapy fractions by treatment on weekends, 34 of 286 patients (11.9\%) in our study eventually experienced a moderate prolongation of the prescribed overall treatment time of 1-5 days. Data concerning the detrimental effect of treatment interruptions in adjuvant breast cancer radiotherapy are scarce. However, a significant decrease in the 5-year local control rate after treatment interruptions of more than 1 week has been reported [26].

\section{Conclusion}

A significant proportion of breast cancer patients in our study experienced treatment interruptions. Compliance with the prescribed radiotherapy schedule was significantly better for patients treated with HF than for those treated with CF. The data suggest that the better compliance is basically related to the shorter overall treatment time of HF (3-4 weeks) compared with CF (5-6.5 weeks). This finding may add to the treatment decision in favor of HF in particular in situations with expected lower compliance with longer radiotherapy schedules.

\section{Compliance with ethical guidelines}

Conflict of interest V. Rudat, A. Nour, M. Hammoud, and S. Abou Ghaida declare that they have no competing interests.

Ethical standards All procedures performed in studies involving human participants were in accordance with the ethical standards of the institutional and/or national research committee and with the 1964 Helsinki declaration and its later amendments or comparable ethical standards. Informed consent was obtained from all individual participants included in the study.

Open Access This article is distributed under the terms of the Creative Commons Attribution 4.0 International License (http:// creativecommons.org/licenses/by/4.0/), which permits unrestricted use, distribution, and reproduction in any medium, provided you give appropriate credit to the original author(s) and the source, provide a link to the Creative Commons license, and indicate if changes were made.

\section{References}

1. Thames HD, Kuban D, Levy LB, Horwitz EM, Kupelian P, Martinez A, Michalski J, Pisansky T, Sandler H, Shipley W, Zelefsky 
M, Zietman A (2010) The role of overall treatment time in the outcome of radiotherapy of prostate cancer: an analysis of biochemical failure in 4839 men treated between 1987 and 1995. Radiotherapy and oncology : journal of the European Society for Therapeutic. Radiol Oncol 96(1):6-12. doi:10.1016/j.radonc.2010.03.020

2. Perez CA, Grigsby PW, Castro-Vita H, Lockett MA (1995) Carcinoma of the uterine cervix. I. Impact of prolongation of overall treatment time and timing of brachytherapy on outcome of radiation therapy. Int J Radiat Oncol Biol Phys 32(5):1275-1288. doi:10. 1016/0360-3016(95)00220-s

3. Fowler JF, Lindstrom MJ (1992) Loss of local control with prolongation in radiotherapy. Int J Radiat Oncol Biol Phys 23(2):457-467

4. Ohri N, Rapkin BD, Guha C, Kalnicki S, Garg M (2016) Radiation therapy noncompliance and clinical outcomes in an urban academic cancer center. Int J Radiat Oncol Biol Phys 95(2):563-570. doi:10. 1016/j.ijrobp.2016.01.043

5. Bese NS, Hendry J, Jeremic B (2007) Effects of prolongation of overall treatment time due to unplanned interruptions during radiotherapy of different tumor sites and practical methods for compensation. Int J Radiat Oncol Biol Phys 68(3):654-661. doi:10.1016/j. ijrobp.2007.03.010

6. Withers HR, Taylor JM, Maciejewski B (1988) The hazard of accelerated tumor clonogen repopulation during radiotherapy. Acta Oncol 27(2):131-146

7. Rudat V, Nour A, Ghaida SA, Alaradi A (2016) Impact of hypofractionation and tangential beam IMRT on the acute skin reaction in adjuvant breast cancer radiotherapy. Radiat Oncol 11(1):100. doi:10.1186/s13014-016-0674-y

8. Rudat V, Nour A, Alaradi AA, Mohamed A, Altuwaijri S (2014) In vivo surface dose measurement using GafChromic film dosimetry in breast cancer radiotherapy: comparison of 7-field IMRT, tangential IMRT and tangential 3D-CRT. Radiat Oncol 9:156. doi:10. 1186/1748-717x-9-156

9. Rudat V, Alaradi AA, Mohamed A, Ai-Yahya K, Altuwaijri S (2011) Tangential beam IMRT versus tangential beam 3D-CRT of the chest wall in postmastectomy breast cancer patients: a dosimetric comparison. Radiat Oncol 6:26. doi:10.1186/1748-717X-626

10. RTOG (2016) Breast Cancer Atlas. https://www.rtog.org/CoreLab/ ContouringAtlases/BreastCancerAtlas.aspx. Accessed 8 Nov 2016

11. Rudat V, Hammoud M, Pillay Y, Alaradi AA, Mohamed A, Altuwaijri $S$ (2011) Impact of the frequency of online verifications on the patient set-up accuracy and set-up margins. Radiat Oncol 6:101. doi:10.1186/1748-717x-6-101

12. Becker-Schiebe M, Stockhammer M, Hoffmann W, Wetzel F, Franz $H$ (2016) Does mean heart dose sufficiently reflect coronary artery exposure in left-sided breast cancer radiotherapy?: Influence of respiratory gating. Strahlenther Onkol 192(9):624-631. doi:10.1007/ s00066-016-1011-y

13. Hepp R, Ammerpohl M, Morgenstern C, Nielinger L, Erichsen P, Abdallah A, Galalae R (2015) Deep inspiration breath-hold (DIBH) radiotherapy in left-sided breast cancer: Dosimetrical comparison and clinical feasibility in 20 patients. Strahlenther Onkol 191(9):710-716. doi:10.1007/s00066-015-0838-y

14. Schonecker S, Heinz C, Sohn M, Haimerl W, Corradini S, Pazos M, Belka C, Scheithauer H (2016) Reduction of cardiac and coronary artery doses in irradiation of left-sided breast cancer during inspiration breath hold : A planning study. Strahlenther Onkol 192(11):750-758. doi:10.1007/s00066-016-1039-z

15. Bahrainy M, Kretschmer M, Jost V, Kasch A, Wurschmidt F, Dahle J, Lorenzen J (2016) Treatment of breast cancer with simultaneous integrated boost in hybrid plan technique : Influence of flattening filter-free beams. Strahlenther Onkol 192(5):333-341. doi:10.1007/ s00066-016-0960-5

16. Jost V, Kretschmer M, Sabatino M, Wurschmidt F, Dahle J, Ueberle F, Lorenzen J (2015) Heart dose reduction in breast cancer treatment with simultaneous integrated boost: Comparison of treatment planning and dosimetry for a novel hybrid technique and 3DCRT. Strahlenther Onkol 191(9):734-741. doi:10.1007/s00066015-0874-7

17. Ott OJ, Strnad V, Stillkrieg W, Uter W, Beckmann MW, Fietkau R (2017) Accelerated partial breast irradiation with external beam radiotherapy : First results of the German phase 2 trial. Strahlenther Onkol 193(1):55-61. doi:10.1007/s00066-016-1066-9

18. Lohr F, El-Haddad M, Dobler B, Grau R, Wertz HJ, Kraus-Tiefenbacher U, Steil V, Madyan YA, Wenz F (2009) Potential effect of robust and simple IMRT approach for left-sided breast cancer on cardiac mortality. Int J Radiat Oncol Biol Phys 74(1):73-80. doi:10. 1016/j.ijrobp.2008.07.018

19. Rudat V, Brune-Erbe I, Noureldin A, Bushnag Z, Almuraikhi N, Altuwaijri S (2012) Epidemiology of breast cancer patients at a tertiary care center in the Eastern Province of Saudi Arabia. Gulf J Oncolog 1(11):45-49

20. Yarnold J, Ashton A, Bliss J, Homewood J, Harper C, Hanson J, Haviland J, Bentzen S, Owen R (2005) Fractionation sensitivity and dose response of late adverse effects in the breast after radiotherapy for early breast cancer: long-term results of a randomised trial. Radiother Oncol 75(1):9-17. doi:10.1016/j.radonc.2005.01.005

21. Owen JR, Ashton A, Bliss JM, Homewood J, Harper C, Hanson J, Haviland J, Bentzen SM, Yarnold JR (2006) Effect of radiotherapy fraction size on tumour control in patients with early-stage breast cancer after local tumour excision: long-term results of a randomised trial. Lancet Oncol 7(6):467-471. doi:10.1016/S14702045(06)70699-4

22. Whelan TJ, Pignol JP, Levine MN, Julian JA, MacKenzie R, Parpia S, Shelley W, Grimard L, Bowen J, Lukka H, Perera F, Fyles A, Schneider K, Gulavita S, Freeman C (2010) Long-term results of hypofractionated radiation therapy for breast cancer. N Engl J Med 362(6):513-520. doi:10.1056/NEJMoa0906260

23. Haviland JS, Owen JR, Dewar JA, Agrawal RK, Barrett J, BarrettLee PJ, Dobbs HJ, Hopwood P, Lawton PA, Magee BJ, Mills J, Simmons S, Sydenham MA, Venables K, Bliss JM, Yarnold JR (2013) The UK Standardisation of Breast Radiotherapy (START) trials of radiotherapy hypofractionation for treatment of early breast cancer: 10-year follow-up results of two randomised controlled trials. Lancet Oncol 14(11):1086-1094. doi:10.1016/S14702045(13)70386-3

24. Shaitelman SF, Schlembach PJ, Arzu I, Ballo M, Bloom ES, Buchholz D, Chronowski GM, Dvorak T, Grade E, Hoffman KE, Kelly P, Ludwig M, Perkins GH, Reed V, Shah S, Stauder MC, Strom EA, Tereffe W, Woodward WA, Ensor J, Baumann D, Thompson AM, Amaya D, Davis T, Guerra W, Hamblin L, Hortobagyi G, Hunt KK, Buchholz TA, Smith BD (2015) Acute and short-term toxic effects of conventionally fractionated vs hypofractionated whole-breast irradiation: a randomized clinical trial. JAMA Oncol 1(7):931-941. doi:10.1001/jamaoncol.2015.2666

25. Jagsi R, Griffith KA, Boike TP, Walker E, Nurushev T, Grills IS, Moran JM, Feng M, Hayman J, Pierce LJ (2015) Differences in the acute toxic effects of breast radiotherapy by fractionation schedule: comparative analysis of physician-assessed and patientreported outcomes in a large multicenter cohort. JAMA Oncol 1(7):918-930. doi:10.1001/jamaoncol.2015.2590

26. Bese NS, Sut PA, Ober A (2005) The effect of treatment interruptions in the postoperative irradiation of breast cancer. Oncology 69(3):214-223. doi:10.1159/000087909

27. Ohri N, Rapkin BD, Guha D, Haynes-Lewis H, Guha C, Kalnicki S, Garg M (2015) Predictors of radiation therapy noncompliance in an urban academic cancer center. Int J Radiat Oncol Biol Phys 91(1):232-238. doi:10.1016/j.ijrobp.2014.09.030

28. Meden T, St. John-Larkin C, Hermes D, Sommerschield S (2002) Relationship between travel distance and utilization of breast cancer treatment in rural northern Michigan. JAMA 287(1):111 
29. Nattinger AB, Kneusel RT, Hoffmann RG, Gilligan MA (2001) Relationship of distance from a radiotherapy facility and initial breast cancer treatment. J Natl Cancer Inst 93(17):1344-1346

30. Athas WF, Adams-Cameron M, Hunt WC, Amir-Fazli A, Key CR (2000) Travel distance to radiation therapy and receipt of radiotherapy following breast-conserving surgery. J Natl Cancer Inst 92(3):269-271
31. Arrossi S, Matos E, Zengarini N, Roth B, Sankaranayananan R, Parkin M (2007) The socio-economic impact of cervical cancer on patients and their families in Argentina, and its influence on radiotherapy compliance. Results from a cross-sectional study. Gynecol Oncol 105(2):335-340. doi:10.1016/j.ygyno.2006.12.010 